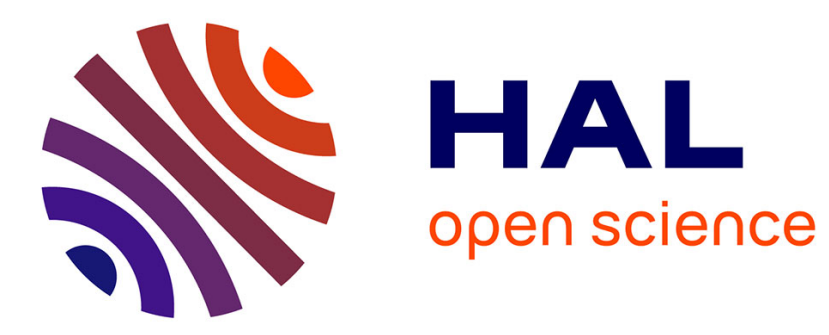

\title{
Recherches archéologiques franco-indonésiennes à Bukit Hasang, Barus, Sumatra-Nord
}

Daniel Perret, Heddy Surachman

\section{To cite this version:}

Daniel Perret, Heddy Surachman. Recherches archéologiques franco-indonésiennes à Bukit Hasang, Barus, Sumatra-Nord. Bulletin de l'Ecole française d'Extrême-Orient, 2002, 89, pp.344-350. 10.3406/befeo.2002.3575 . halshs-01883839

\section{HAL Id: halshs-01883839 \\ https://shs.hal.science/halshs-01883839}

Submitted on 28 Sep 2018

HAL is a multi-disciplinary open access archive for the deposit and dissemination of scientific research documents, whether they are published or not. The documents may come from teaching and research institutions in France or abroad, or from public or private research centers.
L'archive ouverte pluridisciplinaire HAL, est destinée au dépôt et à la diffusion de documents scientifiques de niveau recherche, publiés ou non, émanant des établissements d'enseignement et de recherche français ou étrangers, des laboratoires publics ou privés. 


\section{Recherches archéologiques franco-indonésiennes à Bukit Hasang,}

\section{Barus, Sumatra-Nord}

Daniel Perret, Heddy Surachman

\section{Citer ce document / Cite this document :}

Perret Daniel, Surachman Heddy. Recherches archéologiques franco-indonésiennes à Bukit Hasang, Barus, Sumatra-Nord. In: Bulletin de l'Ecole française d'Extrême-Orient. Tome 89, 2002. pp. 344-350;

doi : https://doi.org/10.3406/befeo.2002.3575

https://www.persee.fr/doc/befeo_0336-1519_2002_num_89_1_3575

Fichier pdf généré le 07/05/2018 


\title{
Recherches archéologiques franco-indonésiennes à Bukit Hasang, Barus, Sumatra-Nord
}

\author{
Daniel PERreT et Heddy Surachman
}

\section{Introduction}

Contrairement à la façade orientale de l'île de Sumatra, qui fait l'objet de nombreuses recherches archéologiques depuis le début du $\mathrm{XX}^{\mathrm{e}}$ siècle, en particulier dans les provinces de Sumatra-Sud et de Jambi, la côte ouest a été pratiquement ignorée des archéologues jusqu'aux années 1980. Cette situation tient probablement à la maigreur des sources anciennes concernant cette zone et à la pauvreté, voire à l'absence, de vestiges monumentaux. La région de Barus, à Sumatra-Nord, surtout réputée de l'Eurasie à la Chine, et ce probablement dès le milieu du premier millénaire au moins, pour ses produits forestiers, en particulier son camphre issu de l'arrière-pays, n'échappe pas à la règle. Si des prospections concernant les tombes anciennes y sont effectuées dès les années 1960 par le Centre national de recherches archéologiques indonésien (Pusat Penelitian Arkeologi Nasional) sous les directions successives de Uka Tjandrasasmita et Hasan M. Ambary, il faut attendre 1978 et surtout 1985 pour assister à la réalisation des premiers sondages. C'est à la même époque qu'on commence à s'intéresser aux textes malais émanant de Barus et aux sources concernant l'histoire de Barus avant le XVI' siècle" ${ }^{11}$. La revue Archipel publie alors les résultats des sondages archéologiques de $1985^{12}$. Ces investigations pionnières prometteuses vont encourager la mise en place d'un programme de recherches archéologiques franco-indonésien, qui se concrétise en 1995 par la première campagne de fouilles sur le site urbain de Lobu Tua, sous la direction de Claude Guillot. Ce programme s'est achevé durant l'année 2000, après cinq campagnes de fouilles et une campagne d'étude de matériel, dont quelques résultats ont déjà été publiés ${ }^{13}$. La richesse d'enseignements tirée de ces fouilles a convaincu le Pusat Penelitian Arkeologi (Centre de recherches archéologiques) et l'École française d'Extrême-Orient de poursuivre des recherches archéologiques à Barus. C'est dans ce but qu'un nouveau programme, bénéficiant du soutien de la Commission consultative des recherches archéologiques à l'étranger du ministère des Affaires étrangères, a été lancé en 2001 avec pour objectif principal l'étude du site urbain de Bukit Hasang. Il vise non seulement à approfondir notre connaissance de l'histoire économique et politique de la région, impossible à reconstituer

11. Quatre publications de Jane Drakard portent sur l'étude des chroniques historiques écrites à Barus et sur l'ensemble des sources disponibles pour l'histoire de Barus : «Ideological Adaptation on a Malay Frontier », Journal of Southeast Asian Studies 17/1 (1986) : 39-57; «An Indian Ocean Port: Sources for the Earlier History of Barus », Archipel 37 (1989) : 53-82 ; Sejarah Raja-Raja Barus : Dua Naskah dari Barus, Jakarta / Bandung, EFEO / Penerbit Angkasa (Textes et documents nousantariens, 7), 1988 ; A Malay frontier: Unity and duality in a Sumatran kingdom, Ithaca, Cornell University, Southeast Asia Program, 1990.

12. Lukman Nurhakim, "La ville de Barus: Étude archéologique préliminaire », Archipel 37 (1989): 43-52.

13. Claude Guillot, Sonny Wibisono, Daniel Perret, « Le programme franco-indonésien de recherche archéologique sur Barus ", Archipel 51 (1996) : 35-45. Claude Guillot (éd.), Histoire de Barus : Le site de Lobu Tua, I. Études et documents, Paris, Association Archipel (Cahiers d'Archipel, 30), 1998. Ce dernier volume a fait l'objet récemment d'une publication en indonésien par le Centre de Jakarta de l'EFEO : Cl. Guillot (éd.), Lobu Tua. Sejarah Awal Barus, Jakarta, EFEO / Association Archipel / Pusat Penelitian Arkeologi / Yayasan Obor, 2002. 
avec les sources actuellement à notre disposition, mais aussi, sur un plan plus général, à apporter une contribution à l'histoire urbaine du monde malais occidental à partir du cas d'un site côtier urbanisé dont l'existence est liée principalement à l'exploitation de ressources naturelles locales. Contemporain des premières traces d'islamisation avérées aujourd'hui en Indonésie, le site de Bukit Hasang pourrait également livrer des données déterminantes sur l'histoire de l'islamisation de cette région. Enfin, avec la redécouverte récente de la stèle funéraire de Hamzah Fansuri ${ }^{14}$, le premier poète et l'un des premiers penseurs soufis du monde malais ${ }^{15}$, qui porte à croire que ce lettré a vécu à Barus au $X V^{e}$ siècle, Bukit Hasang prend une nouvelle dimension comme important creuset de culture malaise. Ce sont les résultats préliminaires des deux premières campagnes de fouilles menées en 2001 et 2002, avec la collaboration du Bureau des recherches archéologiques (Balai Arkeologi) de Medan, qui sont présentés ici.

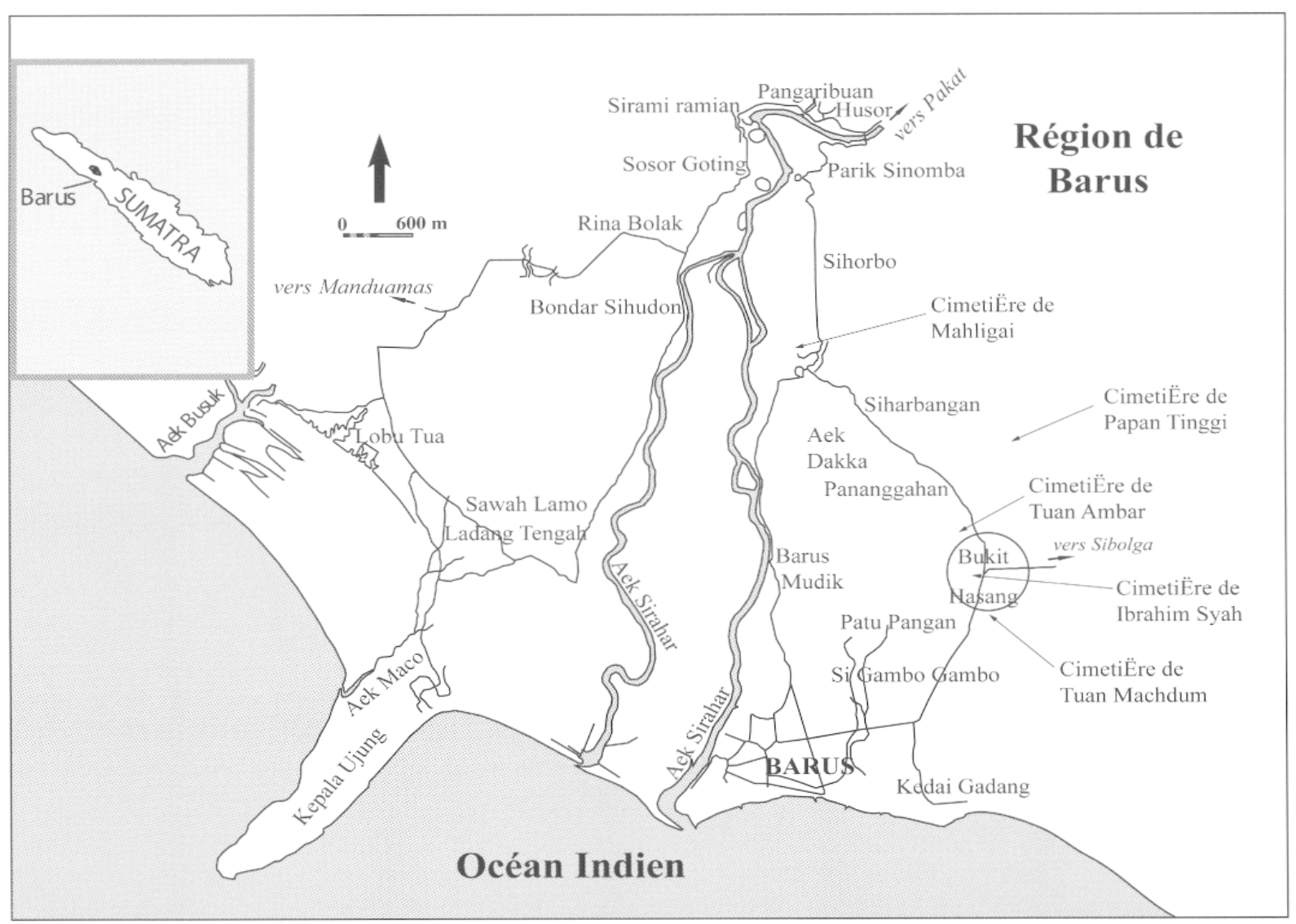

14. Claude Guillot \& Ludvik Kalus, « La stèle funéraire de Hamzah Fansuri », Archipel 60 (2000) : $3-24$.

15. Vladimir I. Braginsky, «Towards the Biography of Hamzah Fansuri. When Did Hamzah Live? Data From His Poems and Early European Accounts », Archipel 57 (1999) : 136. 


\section{Données préalables}

\section{Les données géographiques et historiques}

Situé sur la côte ouest de la province actuelle de Sumatra-Nord, Barus est à la frontière du territoire d'Aceh dans une plaine étroite (de 5 à $15 \mathrm{~km}$ de large) qui s'étend entre l'océan Indien et les contreforts de la haute chaîne montagneuse des Barisan. Barus est aujourd'hui le chef-lieu du kecamatan (canton) portant ce nom dans le kabupaten (département) de Tapanuli Central. Barus a une longue histoire de contacts commerciaux avec le monde extérieur, puisque ce toponyme est cité dès la seconde moitié du premier millénaire dans des sources chinoises et arabes. On le retrouve un peu plus tard dans des sources d'origine tamoule, arménienne, javanaise et malaise. Toutes se limitent néanmoins à faire de Barus un endroit réputé pour le commerce du camphre, et dans une moindre mesure du benjoin, qui attire des marchands originaires de contrées situées entre le Proche-Orient et la Chine. Il faut attendre en fait le début du XVI siècle pour obtenir d'autres informations sur Barus dans les sources écrites étrangères.

Parmi les deux textes locaux publiés en 1988 figure une chronique en malais de la cour du royaume batak de «Barus amont». L'original de la version publiée semble dater de la seconde moitié du XIX ${ }^{e}$ siècle, mais il est clair que le texte reprend des éléments beaucoup plus anciens. S'il est bien sûr impossible de vérifier l'authenticité de tous les détails de cette version, on doit néanmoins admettre que les informations concernant la localisation des sites "urbains " depuis l'origine de Barus, ainsi que le rôle attribué aux communautés étrangères dans le développement commercial initial, se trouvent progressivement confirmées par des données épigraphiques et archéologiques très certainement inconnues du copiste.

Ce texte fournit ainsi une liste des établissements anciens de la région de Barus. Il cite tout d'abord le nom de Aek Busuk Lama, en bordure de la rivière Aek Busuk, puis rapporte le déplacement des habitants vers un endroit appelé Lobu Tua. Plus tard, Lobu Tua est détruit par des « orang Garagasi » (géants ou monstres). Les habitants s'enfuient alors pour fonder Kota Bariang situé sur la rive gauche de la rivière Sirahar. Un second établissement, Gugu', apparaît un peu plus tard en aval. Les habitants de Kota Bariang se déplacent ensuite vers Pintu Ria, alors que ceux de Gugu' se déplacent vers Hujung Tanah. La chronique situe ce dernier événement vers $700 \mathrm{H}$. (soit 1300 E. C.). Les habitants de Pintu Ria s'installent ensuite à Simugari, avant de fonder un nouvel établissement à Lobo Dalam. La région de Barus est plus tard dévastée par une attaque du royaume d'Aceh, situé à la pointe nord de Sumatra. La chronique poursuit en situant l'arrivée des Hollandais en 1640.

Sur le plan religieux, le caractère cosmopolite de Barus, dès ses premiers établissements, incite à penser que plusieurs religions devaient coexister. Les preuves sont malheureusement minces : un fragment de statue de bodhisattva en granit rose trouvé à Lobu Tua et aujourd'hui disparu ; la présence attestée d'une communauté tamoule à Lobu Tua qui devait disposer d'un lieu de culte hindou; enfin, toujours à Lobu Tua, la découverte récente d'un sceau-talisman inscrit en arabe qui pourrait dater du $X^{\mathrm{e}}$ ou du XI siècle. Des sources écrites étrangères signalent la présence de chrétiens nestoriens à Fansur (probablement l'ancien nom de Barus) avant le XIII ${ }^{c}$ siècle, ainsi que le décès d'un marchand juif à Fansur au XIII ${ }^{e}$ siècle. Enfin, les noms de Fansur et Barus sont mentionnés dans les poèmes de Hamzah, le premier poète musulman mystique malais connu, que son nisbah d'al-Fansuri relie à ce lieu et dont on a maintenant la quasi-certitude qu'il a vécu au XV $\mathrm{XV}^{\mathrm{c}}$ siècle à Barus. Cette association avec Hamzah laisse à penser que Barus a pu être un centre islamique d'envergure et également un important creuset de civilisation malaise. 
Les données épigraphiques et archéologiques

Dès les années 1870, des fouilles sauvages menées à Lobu Tua livrent nombre d'objets anciens, y compris des bijoux et des monnaies d'or. Dans le même temps, on y découvre quatre pierres inscrites qui proviennent en fait de deux inscriptions. La première est une inscription en langue tamoule portant une date équivalente à $1088 \mathrm{E}$. C., dont le texte complet ne sera publié qu'en 1998. Euvre d'une guilde de marchands tamouls, son contenu confirme d'une part qu'une colonie de marchands tamouls résidait là de manière plus ou moins permanente, d'autre part que l'endroit était bien connu de ces marchands sous le nom de Barus puisque l'inscription contient la transcription tamoule de ce toponyme. La seconde inscription, composée aujourd'hui de deux fragments, est en javanais ancien postérieur au milieu du $X^{\mathfrak{c}}$ s. E. C. En très mauvais état, elle reste à déchiffrer. Les relations de Barus avec Java sont confirmées par d'autres trouvailles: bagues en or portant des inscriptions en javanais et pièces de monnaie, également en or, de type ma (à fleur de santal).

C'est à partir des années 1960 que les chercheurs du Centre national de recherches archéologiques indonésien ont commencé à s'intéresser aux plusieurs dizaines de tombes musulmanes anciennes de Barus réparties dans cinq complexes. Trois cimetières se trouvent sur le site de Bukit Hasang ou à proximité : Tuan Machdum, Ibrahim Syah et Tuan Ambar. Les deux autres, Papantinggi et Mahligai, se trouvent un peu plus au nord. Plusieurs stèles comportent des inscriptions et selon Ludvik Kalus, qui réalise une étude épigraphique sur ces stèles, la plus ancienne tombe datée pourrait remonter à la fin du XIV siècle.

Les trois sondages et les collectes de tessons en surface effectués en 1985 dans le kecamatan de Barus par Nurhadi et Lukman Nurhakim, du Centre national de recherches archéologiques, avaient pour objectif l'établissement d'une chronologie des sites urbains anciens. Ainsi, quatre sites ont été identifiés et classés chronologiquement comme suit, du plus ancien au plus récent : Lobu Tua, au nord-ouest de la ville actuelle de Barus, Bukit Hasang au nord-est, Kedai Gedang au sud-ouest en bord de mer, et Barus (ou Kampung) Mudik au nord.

Le programme archéologique franco-indonésien qui a démarré en 1995 s'est orienté logiquement vers le site de Lobu Tua, le plus ancien et, a priori, le plus riche en vestiges. Si les limites de l'agglomération ancienne demeurent assez floues, la superficie actuelle de la zone intra-muros, un quadrilatère d'environ 2,5 hectares, est inférieure à la taille du site à l'époque de sa fondation, du fait de l'érosion sur trois de ses côtés. À l'issue de cinq campagnes de fouilles menées entre 1995 et 1999, un matériel abondant a été collecté. L'examen de ce matériel indique que la période d'occupation du site de Lobu Tua fut courte, environ deux siècles, de la fin du $\mathrm{IX}^{\mathrm{e}}$ siècle au tournant du XII ${ }^{\mathrm{e}}$. Cette occupation cessa brusquement, puisque pratiquement aucun matériel postérieur à cette période n'a été collecté. Le matériel archéologique est par conséquent d'une rare homogénéité et confirme par ailleurs l'existence de relations avec une zone s'étendant du Proche-Orient à la Chine en passant par l'Iran, l'Inde et l'archipel insulindien.

\section{Les premières fouilles de Bukit Hasang}

\section{Problématique}

Le lancement du programme de fouilles à Bukit Hasang s'est appuyé sur les acquis des fouilles de Lobu Tua, en particulier une chronologie bien établie, ainsi que sur les résultats de deux sondages réalisés à Bukit Hasang, l'un en 1985, l'autre en 1997. Si les résultats de ces deux derniers sondages s'accordaient bien sur le caractère plus récent du 
site, ils divergeaient sur le début de son occupation. Le premier semblait en faire la suite directe de Lobu Tua, alors que le second indiquait un intervalle temporel assez long entre l'abandon de Lobu Tua et le début de Bukit Hasang.

Le programme de fouilles de Bukit Hasang tente d'éclairer quatre aspects : chronologie précise du site, origine et mode de vie des habitants, structure et organisation du site. À la richesse d'enseignements que doit procurer la fouille pour l'histoire du site même s'ajoute ici la possibilité d'une étude comparative avec celui de Lobu Tua. Nous espérons également que des indices permettront de cerner les rapports de Bukit Hasang avec les autres comptoirs ou centres politiques et économiques contemporains de l'île, tels que Padang Lawas (un grand complexe archéologique dans l'intérieur de la province), la pointe nord de Sumatra, les comptoirs et centres du détroit de Malacca (Pasai, Kota Cina, Jambi, etc.) ou plus loin avec Java, l'océan Indien, la Chine et le Proche-Orient. Sur le plan religieux et culturel, la combinaison des données épigraphiques tirées des stèles de Bukit Hasang et des données archéologiques provenant des fouilles devrait apporter des informations déterminantes sur l'histoire de l'islamisation et de la civilisation malaise dans la région.

\section{Les fouilles}

Le site de Bukit Hasang (centre du site, secteur B : 02 $011^{\prime} 44^{\prime \prime}$ N ; 98 $24^{\prime} 51^{\prime \prime}$ E) se trouve sur la bordure ouest d'un contrefort de la chaîne des Bukit Barisan, qui surplombe d'une dizaine de mètres la plaine en direction de l'océan Indien, dont les rives sont à environ $3 \mathrm{~km}$. Ce contrefort se prolonge vers le nord jusqu'à la rivière Sirahar, alors que vers le sud il prend la forme d'un promontoire allongé se rétrécissant progressivement pour disparaître dans des rizières à environ $1 \mathrm{~km}$ de la côte. De grandes zones de rizières sont exploitées à l'est et à l'ouest du site, que la route Barus-Pakat/Manduamas coupe pratiquement en deux du nord au sud. Le village actuel de Bukit Hasang, qui compte plus de 1000 habitants, occupe tout le site. L'habitat est dense, surtout en bordure des routes Barus-Pakat et Barus-Sibolga. Les parcelles inexploitées sont très rares, la plupart étant plantées de cacaoyers, cocotiers et divers arbres fruitiers. Le choix des lieux de sondages a dû se faire en tenant compte du couvert végétal, de façon à endommager un minimum d'arbres. Il a également été fait en fonction des autorisations données par les nombreux propriétaires, car beaucoup de parcelles sont exploitées en indivision.

La campagne 2001 (5 août au 16 septembre) s'est donné comme objectifs une prospection systématique sur la commune de Bukit Hasang et la réalisation de plusieurs sondages sur cette même commune, afin d'évaluer le potentiel du site, de préciser ses limites spatiales et d'obtenir des informations sur la chronologie de son occupation. Mis à part les deux cimetières musulmans anciens de Tuan Ibrahim Syah (approximativement au centre du site) et Tuan Machdum (à une centaine de mètres au sud du site principal), plusieurs tombes anciennes isolées ont été repérées dès cette première campagne. La prospection initiale a permis également de constater que des tessons de poteries et de céramiques étaient visibles en surface (avec une densité qui semble décroître en direction de l'est) sur une superficie estimée provisoirement à 10 ha (longueur d'environ $600 \mathrm{~m}$ direction nord-sud - et largeur entre 100 et $200 \mathrm{~m}$ ), ce qui représente quatre fois la superficie du site intra-muros de Lobu Tua. Ce calcul ne tient pas compte de la partie qui a disparu par érosion de la pente à l'ouest.

Onze secteurs ont été fouillés en 2001. Dans chaque secteur, un nombre variable de carrés de sondage $(2,5 \mathrm{~m} \times 2,5 \mathrm{~m})$ ont été creusés. 39 carrés ont ainsi été ouverts, dont 35 en totalité. 41 carrés ont été fouillés en totalité ou partiellement lors de la campagne 2002, qui s'est déroulée du 7 avril au 18 mai. Les sondages ont commencé à l'ouest de la route Barus-Pakat, donc en bordure du plateau, car c'est là qu'on peut observer les plus grandes 
densités de tessons en surface, ainsi que plusieurs monticules laissant supposer l'existence de structures. Ce sont précisément les sondages effectués au voisinage des monticules qui se sont révélés les plus intéressants.

\section{Secteur B}

Au centre du site, le secteur B comprend le point le plus élevé du site au sommet d'un monticule de forme irrégulière, d'environ deux mètres de hauteur. Deux carrés ouverts sur les flancs de ce monticule ont livré une structure comprenant des blocs de madrépore.

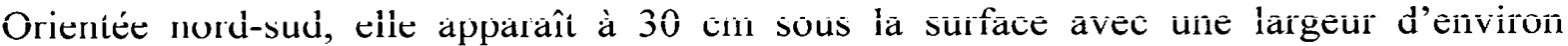
$1,70 \mathrm{~m} .11$ nouveaux carrés ont été ouverts dans ce secteur en 2002 pour essayer de déterminer sa forme. Bien qu'elle ait pratiquement disparu dans les carrés les plus au nord du secteur, il est clair qu'on la retrouve selon un axe nord-sud sur une distance minimale de 45 mètres. Le démontage partiel de cette structure a permis d'observer qu'elle se compose d'une tranchée d'une largeur maximale de $3 \mathrm{~m}$ et d'une profondeur de $30 \mathrm{~cm}$ environ. Cette tranchée a été remblayée avec de la terre surmontée d'un mélange de blocs de corail (environ $80 \%$ ), de galets et de terre sur une épaisseur d'environ $60 \mathrm{~cm}$. De plus, l'un des carrés fouillés en 2001 présente dans la tranchée une fosse d'une profondeur maximale de $1,60 \mathrm{~m}$, qui s'étend sous le mur en corail et a livré environ $10 \mathrm{~kg}$ d'os de divers animaux. Les habitants de Bukit Hasang affirment d'une part que le madrépore n'a jamais fait partie des matériaux utilisés depuis la fondation du village actuel (il y environ 150 ans), d'autre part que les coutumes locales n'ont jamais prescrit de sacrifices d'animaux dont les carcasses seraient ensuite déposées sous une construction. Il y a par conséquent tout lieu de penser que l'édification de cette structure remonte à la période d'occupation initiale du site. Une tranchée de sondage de $12 \mathrm{~m}$ réalisée du côté ouest de la structure montre une série de fosses relativement riches en matériel. La présence de ces fosses pourrait indiquer l'existence d'un habitat dense dans le secteur. La fonction de cette structure reste énigmatique. Le fait qu'on retrouve des fragments de corail ailleurs sur le site, à peu près dans le même axe nord-sud, pourrait faire penser à un mur séparant le site en deux grands quartiers. De nouveaux sondages seront nécessaires pour tenter de confirmer cette hypothèse.

\section{Secteur A}

Sept carrés ont été ouverts en 2001 dans le secteur A, qui se trouve dans la partie nord du site. Ce secteur est remarquable par un monticule en forme de quadrilatère (environ $10 \mathrm{~m}$ de côté), d'une hauteur de $3 \mathrm{~m}$ environ au sommet. Sur ce monticule se trouve une pierre carrée $(140 \times 140 \times 28 \mathrm{~cm})$ sculptée d'un sillon central sur toute sa longueur. Ces éléments suggèrent l'existence d'un lieu de culte ancien. La stratigraphie de la base du monticule semble indiquer qu'il s'agit d'une structure artificielle. Un puits ancien de cinq mètres de profondeur a été découvert lors de sondages réalisés à quelques mètres du monticule. Il a livré notamment plusieurs poteries intactes ou presque, en forme de « jarrebouteille » qui semblent exceptionnelles en Indonésie.

\section{Secteur M}

L'un des aspects de la campagne 2002 a été de tenter de déterminer la limite est du site principal. Une légère dépression visible au nord-est pouvait laisser supposer l'existence d'une douve, comme c'est le cas sur le site de Lobu Tua. Une tranchée de sondage coupant cette dépression montre qu'il s'agit en fait d'un cours d'eau de faible profondeur aujourd'hui remblayé. Il marque bien la limite du site principal, car les sondages réalisés sur sa rive est n'ont pour l'instant livré pratiquement aucun matériel, alors que ceux réalisés sur sa rive ouest ont une densité de matériel équivalente à celle des 
sondages effectués en 2001. Cette hypothèse sur l'absence d'ouvrage défensif demande toutefois à être confirmée par la poursuite de sondages au sud-est du site, sondages malheureusement interrompus en 2002 en raison des intempéries.

Dans ce qui peut, très probablement, être considéré comme le cœur du site de Bukit Hasang, l'épaisseur de la couche anthropisée varie entre $10 \mathrm{~cm}$ et $1 \mathrm{~m}$ à partir de la surface. La densité de l'habitat et l'intensité des activités agricoles rendent par conséquent assez peu probable la possibilité de dégager des niveaux non bouleversés. Les seuls faits archéologiques observés dans la plupart des secteurs sont des fosses de terre noire, de taille et de profondeur diverses, souvent riches en matériel.

Six sondages réalisés en 2001 et 2002 dans le secteur G, au pied du site, côté ouest, se sont révélés exceptionnellement riches en matériel. Ils ont livré en fait près de la moitié des tessons de poteries et de céramiques collectés durant ces deux premières campagnes. Cette collecte permet d'apprécier la diversité du matériel présent sur le site avec un minimum de sondages, évidemment hors de tout contexte stratigraphique.

$\mathrm{Au}$ total, les 80 sondages réalisés au cours des campagnes de fouilles 2001 et 2002 ont livré notamment plus de 90000 tessons de poteries représentant un poids proche d'une tonne, environ 34000 tessons de céramiques importées, environ 900 tessons de verre du Proche-Orient et une vingtaine de monnaies ou fragments de monnaies chinoises. Par comparaison, les 172 carrés fouillés lors des cinq campagnes réalisées à Lobu Tua entre 1995 et 1999 ont livré $860 \mathrm{~kg}$ de poteries, 17000 tessons de céramiques et 9000 tessons de verre du Proche-Orient. À noter que la quantité importante de gros tessons de poteries, qui présentent des formes et des décors très divers, permet d'envisager une étude d'un grand intérêt sur ce type de matériel à Bukit Hasang.

Afin de pouvoir commencer à cerner la chronologie du site, l'étude des tessons de céramiques importées trouvés en 2001 a été effectuée par Marie-France Dupoizat au cours de la campagne 2002. Il ressort de cette étude une forte homogénéité chronologique, puisque la très grande majorité des tessons sont datés de la fin du XIII ${ }^{\mathrm{e}}$ siècle au XIV ${ }^{\mathrm{e}}$ siècle, par conséquent de l'époque Yuan. Si cette étude confirme bien que Bukit Hasang est postérieur à Lobu Tua, elle montre également l'existence d'un fossé chronologique de 150 ans environ entre l'abandon de Lobu Tua et le début de l'occupation de Bukit Hasang. Cette conclusion provisoire recoupe sur deux points la chronique locale ancienne. En effet, si cette dernière ne cite pas nommément Bukit Hasang, elle mentionne l'installation de populations à Pintu Ria postérieurement à l'abandon de Lobu Tua. Or le toponyme Pintu Ria existe toujours et marque en fait l'entrée nord du site principal de Bukit Hasang. Les fouilles confirment donc l'existence du site urbain ancien indiqué dans la chronique. Par ailleurs, le fossé chronologique va également dans le sens de la chronique, qui mentionne l'occupation du site de Kota Bariang entre l'abandon de Lobu Tua et l'ouverture de Pintu Ria/Bukit Hasang. Ce site de Kota Bariang a pour l'instant échappé à toutes les investigations archéologiques. Le second enseignement important que l'on peut tirer de la datation proposée par Marie-France Dupoizat est la brièveté de l'occupation de Bukit Hasang, ce qui permettra de dater d'autant plus précisément le reste du matériel, notamment les poteries et le verre. Si elles sont confirmées, ces datations précises seront d'un grand intérêt pour aider à la datation du matériel d'autres sites indonésiens, voire asiatiques, qui n'ont pas connu une période d'occupation aussi courte. 